disease, myocardial ischemia, pulmonary hypertension) was evaluated by 2D transthoracic echocardiography (TTE).

Statistical analysis was done in SPSS, $\mathrm{p}<0.05$.

Results Valvular disease was reported in 61.98\% SLE (14\% stenosis, 20.66\% valvular masses, 33\% mild-to- moderate regurgitation, $34.73 \%$ leaflet thickening. Libman-Sacks endocarditis was not depicted. Asymptomatic decrease in left ventricular ejection fraction (55\%) was described in $16.52 \%$, the lowest LVEF being $33 \%$ in $4.13 \%$ patients; statistical significant negative correlation LVEF - disease duration and activity $(p<0.05)$ was found. LV diastolic dysfunction as subclinical cardiac involvement was registered in 59.50\% SLE, with a direct correlation with disease duration $(\mathrm{p}<0.05)$, but not with disease activity (SLEDAI) and organ damage (SLIC/ACR) $(p>0.05)$. Global hypokinesis on TTE as an indicator of subclincal myocarditis was demonstrated in up to one third SLE, while cardiomyopathy in one fourth. Mild pericardial effusion is observed in $31.2 \%$ cases, while cardiac tamponade in 4 cases; pericardial thickening was reported in 38\% SLE. Finally, abnormal systolic pressure in pulmonary artery was found in $24.79 \%$ patients.

Conclusion Patients with SLE are at increased risk to develop either clinical or subclinical cardiovascular manifestations as demonstrated by echocardiographic studies. A systematic TTE assessment is routinely recommended for the screening and monitoring of cardiac events.

\section{PS1:19 BIOLOGICAL DMARDS-INDUCED LUPUS IN PATIENTS WITH RHEUMATOID ARTHRITIS: A SINGLE CENTRE EXPERIENCE}

${ }^{1} \mathrm{C}$ Ancuta, ${ }^{1,2} \mathrm{C}$ Pomirleanu, ${ }^{2} \mathrm{R}$ Paiu, ${ }^{1} \mathrm{C}$ lordache, ${ }^{3} \mathrm{E}$ Ancuta, ${ }^{4} \mathrm{R}$ Chirieac. ${ }^{1}$ University of Medicine and Pharmacy Grigore T Popa, lasi, Romania; ${ }^{2}$ Clinical Rehabilitation Hospital, Rheumatology Department, lasi, Romania; ${ }^{3}$ Elena Doamna Clinical Hospital, lasi, Romania; ${ }^{4}$ Sanocare Medical and Research Centre, lasi, Romania

\subsection{6/lupus-2018-abstract.67}

Biological drugs (bDMARDs) have changed landscape and outcomes in various rheumatic conditions. Increased risk for infections, paradoxical reactions, autoimmunogenicity are widely acknowledged and may be responsible for treatment failure and switching among biologics.

Objective To assess drug-induced autoimmunity (serology, clinical significance) in patients with rheumatoid arthritis (RA) treated with bDMARDs.

Patients and methods Longitudinal observational study in 246 bionaïve RA receiving their first bDMARD, anti-tumour necrosis factor (TNF) or non-TNF drugs, according to local treatment guidelines.

Disease activity, therapeutic response and serial autoantibody profiles (antinuclear (ANA), anti-dsDNA) were systematically assessed at baseline and every six months. Prevalence, clinical significance and therapeutic implications of ANA/antidsDNA specificities were further analysed (SPSS-19, $\mathrm{p}<0.05$ ).

Results 214 RA received TNF inhibitors (56 infliximab, 65 adalimumab, 8 golimumab, 18 certolizumab, 67 etanercept) as first bDMADs and 32 different mechanism of action (10 rituximab, 7 abatacept, 15 tocilizumab). Overall, ANA seroconversion was demonstrated in $14.22 \%(35 / 246)$, while anti-dsDNA in $8.94 \%$ $(22 / 246)$; no change in the immunological profile under golimumab, certolizumab, abatacept or rituximab.
Significantly higher rates of ANA were reported in infliximabtreated RA (30.53\%) as compared to adalimumab (18.46\%), etanercept $(7.46 \%)$ and tocilizumab (6.66) $(\mathrm{p}<0.05)$; anti-dsDNA positivity was found in 10 infliximab (17.85\%), 4 adalimumab (12.30\%), 3 etanercept $(4.47 \%)$ and one tocilizumab (6.66\%), within an average of 36.5 (2-67) months.

ANA/anti-dsDNA positive status correlated with RA activity (DAS28-ESR) and loss of therapeutic response, concomitant DMARDs (leflunomide) and corticosteroids, administration adverse reactions $(\mathrm{p}<0.05)$.

Only four patients $(1.62 \% ; 4 / 246)$ were classified as druginduced lupus. Three of them (infliximab, adalimumab, tocilizumab -induced) developed mild to moderate disease, with characteristic malar rash, serositis, haematological, elevated ANA and anti-dsDNA that normalised six months after bDMARD discontinuation; the fourth patient had adalimumabinduced lupus two months after drug initiation, with severe course (subacute extensive skin lesions, cytopenia, serositis) and fatal outcome.

Conclusions Development of ANA with or without antidsDNA specificity is typically associated with loss of response to bDMARD in RA and infrequently responsible for druginduced lupus. Systematic evaluation of ANA/dsDNA is not recommended in routine practice, but essential in certain specific clinical settings (secondary non-responders or lupus-like symptoms).

\section{PS1:20 MINING FOR COMMON REACTIVITY PATTERNS OF HUMAN AUTOANTIBODIES AGAINST ENDOGENOUS PROTEIN TARGETS USING CLUSTERED AUTOANTIBODY REACTIVITIES}

H-D Zucht, P Budde, L Steeg, M Tuschen, S Bhandari, P Schulz-Knappe. Protagen AG, Dortmund, Germany

\subsection{6/lupus-2018-abstract.68}

Purpose Autoimmune diseases arise from an abnormal immune response of the body against self-proteins leading to tissue and organ damage. The excessive production of harmful autoantibodies ( $\mathrm{AAB})$ is a hallmark of autoimmune diseases including rheumatoid arthritis (RA), systemic lupus erythematosus (SLE), systemic sclerosis (SSc) and Sjogren's syndrome (SjS). Also in cancer research, it has been recently shown that AABs are useful to characterise patients. The characterisation of patient subgroups by means of stratification is essential for the efficient development of therapies, but often difficult due to the lack of appropriate biomarkers. Personalised or precision medicine approaches rely on appropriate multivariate multiplexing technology and data analysis. AABs serve as diagnostic markers for various autoimmune diseases, but the co-occurrence of $\mathrm{AABs}$ has rarely been analysed and is difficult to comprehend. Detecting a broad set of AABs helps to investigate the similarity between patients. A multiplex signature enables clustering for the investigation of relationships and patterns, which can be related to relevant clinical variables.

Methods Here, we illustrate Luminex bead-based AAB assays using a set of 96 biomarker targets and their utility to characterise SLE and SSc as well as cancer study groups.

Data analysis is based on bi-clustering algorithms and a prevalence and signature analysis of the markers. Cluster analysis was also performed using transformed data sets (qualitative) to 
investigate and visualise characteristic marker prevalence and coprevalence patterns.

Results Based on the individual marker pattern, patients can often be stratified belonging to different study subgroups. For example, for SLE we show that different reactivity groups exist including patients with different disease activity scores and organ damage patterns.

Conclusions We conclude that the approach of a comprehensive prevalence and signature analysis and a vivid data visualisation is useful for any multiplex omics assay.

\section{PS1:21 URINARY MARKERS OF INFLAMMATION IN LUPUS NEPHRITIS PATIENTS}

J Kosalka, B Jakiela, J Musial. Jagiellonian University Medical College, Department of Medicine, Krakow, Poland

\subsection{6/lupus-2018-abstract.69}

Objectives Renal involvement is the most important manifestation of systemic lupus erythematosus, but assessing of inflammatory response in kidneys with non-invasive methods is still challenging. In this study we aimed to define markers of active lupus nephritis (LN) using urine immune profiling.

Methods Levels of cytokines (18-plex array) and mRNA expression (40 immune and glomerular injury genes) were measured in urine samples of $\mathrm{LN}$ patients with active disease $(\mathrm{n}=17)$, during remission $(\mathrm{n}=16)$, and in healthy subjects $(\mathrm{n}=19)$.

Results Urine levels of CCL2, CCL5, CXCL10 and IL-6 were elevated in active LN as compared to remission (best discrimination for CCL2), and correlated with LN activity. In the active disease, urinary cell transcriptome showed strong upregulation of proinflammatory cytokines (e.g. TNF, CCL2, CCL5, CXCL10), Th1 related genes (e.g. CD3G, CD4, TBX21, IFNG), and markers of glomerular damage (NPHS2 [podocin]). Active pattern of gene expression was also observed in 5 patients in remission, who had moderately increased urinary leukocyte count, two patients from this group (40\%) developed renal exacerbation during following 3 months. Markers of Th17 immune axis (e.g. IL-17A) were not significantly increased in active LN.

Conclusions Active LN patients (also patients at risk of exacerbation) were characterised by marked increase of proinflammatory mediators in the urine. We identified CCL2 chemokine as the most promising marker for monitoring of disease flare.

\section{PS1:22 USE OF INTERFERON ALPHA AND INTERLEUKIN-10 AS CLINICAL ACTIVITY BIOMARKERS IN SYSTEMIC LUPUS ERYTHEMATOUS PATIENTS}

${ }^{1} \mathrm{E}$ Grau, ${ }^{2} \mathrm{M}$ Fernandez Matilla, ${ }^{1} \mathrm{CM}$ Feced Olmos, ${ }^{1} \mathrm{E}$ Labrador Sanchez, ${ }^{1} \mathrm{FM}$ Ortiz Sanjuan, ${ }^{2} \mathrm{~N}$ Fernandez-Llanio Cornella, ${ }^{1} \mid \mathrm{C}$ Chalmeta Verdejo, ${ }^{1} \mathrm{~K}$ Arevalo Ruales, ${ }^{1} \mathrm{R}$ Negueroles Albuixech, ${ }^{1} \mathrm{~J}$ Ivorra Cortes, ${ }^{1} \mathrm{JJ}$ Fragio Gil, ${ }^{1} \mathrm{I}$ Martinez Cordellat, ${ }^{1} \mathrm{R}$ Gonzalez Mazario, 'L Gonzalez Puig, ${ }^{1}$ C Alcañiz Escandell, ${ }^{1} \mathrm{C}$ Najera Herranz, 'I Canovas Olmos, ${ }^{1} E$ Vicens Bernabeu, ' JE Oller Rodriguez, ${ }^{1} \mathrm{M}$ de la Rubia Navarro, ${ }^{2} \mathrm{JA}$ Castellano Cuesta, ${ }^{3} \mathrm{~V}$ Fornes Ferrer, ${ }^{3} \mathrm{D}$ Hervas Marin, ${ }^{1} \mathrm{JA}$ Roman Ivorra. ${ }^{1}$ Rheumatology Department. HUP La $\mathrm{Fe}$, Valencia, Spain; ${ }^{2}$ Rheumatology Section. Hospital Arnau de Vilanova, Valencia, Spain; ${ }^{3}$ Biostatistics Unit. IIS La Fe, Valencia, Spain

\subsection{6/lupus-2018-abstract.70}

Purpose To analyse the association among INF1A, IL10 and BLyS levels and clinical activity in SLE.
Methods A cross-sectional, observational study of 142 patients diagnosed of SLE according to SLICC 2012 criteria and 34 healthy controls was performed. In patients a complete bloodtest was made, and clinical data by personal interview was collected. We analysed the serum concentration of IL10, BLyS and INF1A by colorimetric methods. SLE patients were dichotomized as high and low levels for each cytokine based on the cytokine level above $2 \mathrm{SD}$ of the mean in healthy controls. Biostatistical analysis with R (3.3.2.) was performed.

Results In our SLE patients we observed higher values of IL10, BLyS and INF1A than healthy controls $(p<0.001$, $\mathrm{p}=0.005$ and $\mathrm{p}=0.043$ respectively), showing an average values in patients of $13.39 \pm 27.73 \mathrm{pg} / \mathrm{mL}$ INF1A, $9.99 \pm 15.84 \mathrm{pg} / \mathrm{mL}$ IL10 and $1811.31 \pm 1757.81 \mathrm{pg} / \mathrm{mL}$ BLyS. The mean clinical activity measured by SLEDAI was $5.91 \pm 5.06$.

Statistical analysis indicate that INF1A levels are correlated to IL10 levels $(p=0.001)$ and BLyS levels $(p=0,034)$. Due to this finding, we categorised SLE patients by low or high level of the three cytokines: 44 INF1A(-)IL10(-)BLyS(-); 61 INF1A (+)IL10(-)BLyS(-); 5 INF1A(+)IL10(-)BLyS(+); 18 INF1A(+) $\operatorname{IL} 10(+) \mathrm{BLyS}(-)$ and $14 \operatorname{INF} 1 \mathrm{~A}(+) \operatorname{IL} 10(+) \mathrm{BLyS}(+)$. There is a high association of increased IL10-INF1A levels and the increased of clinical activity measured by SLEDAI score $(\mathrm{p}<0.0001)$, and to a lesser extent with increased IL10INF1A-BLyS levels. Patients with high IL10-INF1A and IL10INF1A-BLyS showed a significant rise in C3-C4 consumption $(p<0.001$ and $p=0.001$ respectively) and high anti-dsDNA $(p=0.001$ and $p=0.002$ respectively). Patients with increased INF1A-BLyS exhibited high anti-dsDNA $(p=0.004)$ and ENA positivity $(p<0.001)$. In addition, patients with increased levels of IL10-INF1A-BLyS showed ANAs $(\mathrm{p}<0.001)$ and antiphospholipid autoantibody positivity $(\mathrm{p}=0.004)$.

Conclusions The $69 \%$ of our SLE patients displayed almost one cytokine increased, being the INF1A the cytokine that mainly is increased. However, increased IL10 levels, irrespective of whether there is also increased levels of BLyS and/or INF1A, is the cytokine which best fits to clinical activity in SLE.

\section{Poster session 2: Autoantibodies, biomarkers and imaging (2), Environmental, epigenetics and genomics}

\section{PS2:23 ANTI-C1Q ANTIBODIES IN TURKISH SYSTEMIC LUPUS ERYTHEMATOSUS PATIENTS}

S Tekeoglu, D Temiz Karadag, O Ozdemir Isik, A Yazici, A Cefle. Kocaeli University, Internal Medicine, Rheumatology Department, Kocaeli, Turkey

\subsection{6/lupus-2018-abstract.71}

Purpose Anti-C1q has been shown to be associated with systemic lupus erythematosus (SLE) and disease activity of lupus nephritis in previous studies. We studied anti-C1q specificity for SLE versus rheumatic disease controls and healthy controls and the association with SLE manifestations in a single centre cross-sectional study.

Methods Demographics, disease information and blood samples were obtained during routine follow-up visits of patients attending Kocaeli University rheumatology outpatient clinic. There were 150 SLE patients (92\% female, mean: 46 years). Control group had 85 rheumatoid arthritis patients, 16 